\title{
Costos de atención médica atribuibles al tabaquismo en el IMSS, Morelos
}

\author{
Luz Myriam Reynales-Shigematsu, MD, M en C,(1) Sergio Arturo Juárez-Márquez, MD,(2) \\ Raydel Valdés-Salgado, $M$ en $C^{(1)}$
}

\author{
Reynales-Shigematsu LM, Juárez-Márquez SA, \\ Valdés-Salgado R. \\ Costos de atención médica atribuibles al \\ tabaquismo en el IMSS, Morelos. \\ Salud Publica Mex 2005;47:45 I-457.
}

\begin{abstract}
Resumen
Objetivo. Estimar los costos de atención médica en el Instituto Mexicano del Seguro Social (IMSS), Delegación Morelos, de las enfermedades mayores atribuibles al consumo de tabaco. Materiales y métodos. Las estimaciones de costos se realizaron desde la perspectiva del proveedor utilizando la metodología de costeo de enfermedad. Un panel de expertos multidisciplinario caracterizó la atención médica, en términos de la frecuencia de utilización de servicios en el primer y segundo niveles de atención, considerando el grado de severidad de la enfermedad. Los costos unitarios se estimaron en pesos mexicanos del 200I. Para estimar los costos atribuibles al tabaquismo se utilizó la fracción atribuible al consumo de tabaco para cada enfermedad (FA). Resultados. Los costos promedio anuales de atención médica en el primer y segundo niveles de atención en la Delegación Morelos corresponden a 79530 pesos para infarto agudo del miocardio (IAM), 73303 pesos para enfermedad pulmonar obstructiva crónica (EPOC) y 102215 pesos para cáncer de pulmón (CP). El costo total anual para la Delegación por estas tres enfermedades asciende a 147 millones 390 mil 688 pesos. Los costos atribuibles al consumo de tabaco corresponden a 124 millones de pesos, lo cual equivale a $7.3 \%$ del presupuesto anual de la Delegación. Conclusiones. Estos resultados confirman el alto costo de la atención médica en el IMSS correspondiente a las enfermedades atribuibles al consumo de tabaco. Se recomienda realizar este estudio en el plano nacional, de tal manera que los tomadores de decisiones tengan herramientas para fortalecer las políticas de control del tabaquismo en México.
\end{abstract}

Palabras clave: costos de atención médica; tabaquismo; infarto del miocardio; enfermedad pulmonar obstructiva crónica; cáncer de bronquios y pulmón; México
Reynales-Shigematsu LM, Juárez-Márquez SA, Valdés-Salgado $\mathbf{R}$.

Costs of medical care attributable to tobacco consumption at the Mexican Institute of Social Security (IMSS), Morelos. Salud Publica Mex 2005;47:45 I-457.

\begin{abstract}
Objective. To estimate the cost of medical care for the major diseases attributable to tobacco consumption at the IMSS, Morelos. Material and Methods. A cost of illness (COI) analysis was carried out from the perspective of the health provider. An expert panel characterized medical care in primary and secondary care levels according to severity of disease.The smoking attributable fraction (SAF) by disease was used to derive costs attributable to tobacco consumption. The unitary cost was valuated in 200I Mexican pesos (MP). Results. The estimated annual average cost of medical care (diagnosis and first year of treatment) was 79530 MP for acute myocardial infarction (AMI); 73303 MP for chronic obstructive pulmonary disease (COPD); and 102215 MP for lung cancer (LC). The annual total cost of medical care for IMSS was I47 390688 MP.The total annual cost of medical care attributable to tobacco consumption corresponds to $\$ 124$ million MP, which is equivalent to $7.3 \%$ of the annual budget of the Morelos Delegation. Conclusions. These results confirm the high medical costs associated with smoking. A repetition of this study at the national level is recommended in order to support decision-makers in strengthening public policies to control tobacco use in Mexico.
\end{abstract}

Key words: smoking; health care costs; myocardial infarction; chronic obstructive pulmonary disease; lung cancer; Mexico

(I) Centro de Investigación en Salud Poblacional, Instituto Nacional de Salud Pública, Cuernavaca, Morelos, México.

(2) Coordinación de Investigación Médica, Delegación Morelos, Instituto Mexicano del Seguro Social. Grant CONACyT: SEP-CONACyT 2002-I 40593 , Grant Fogarty: ROI-HL-73699.

Fecha de recibido: 25 de mayo de 2005 - Fecha de aprobado: 12 de octubre de 2005

Solicitud de sobretiros: Luz Myriam Reynales-Shigematsu. Instituto Nacional de Salud Pública. Av. Universidad N ${ }^{\circ} 655$, colonia Santa María Ahuacatitlán, 62508 Cuernavaca, Morelos. México.

Correo electrónico: Ireynales@correo.insp.mx 
$\mathbf{E}$ xiste suficiente evidencia epidemiológica que demuestra que el tabaco es la principal causa modificable de más de 25 enfermedades, entre ellas diferentes tipos de cáncer, enfermedades cardiovasculares y respiratorias. ${ }^{1-6}$ El tabaquismo se ha convertido en un problema de salud pública prioritario en el plano mundial, ya que de los 1100 millones de fumadores en el mundo, 800 millones pertenecen a países en desarrollo. ${ }^{7}$

De acuerdo con la Encuesta Nacional de Adicciones (ENA) del año 2002, realizada en población urbana de 12 a 65 años, la prevalencia de fumadores en México es de $26.4 \%$; esto corresponde a una población de 14 millones de mexicanos fumadores. ${ }^{8}$ Anualmente ocurren cerca de 25383 muertes atribuibles al consumo de tabaco, de las cuales 5165 son por neoplasia, 9057 por enfermedades respiratorias, 5345 por enfermedades cardiovasculares y 5816 por otras causas médicas. ${ }^{9,10}$

El peso económico que estas enfermedades imponen a la sociedad se relaciona con los gastos médicos de la enfermedad y sus complicaciones, las pérdidas de productividad y los años potenciales de vida perdidos por incapacidad o muerte prematura. ${ }^{11-15}$ Estimaciones realizadas en los países desarrollados refieren que los costos de atención médica atribuibles al consumo de tabaco oscilan entre 0.10 y $1.1 \%$ del Producto Interno Bruto (PIB). En los países de bajos y medianos ingresos, aunque con datos muy limitados, las estimaciones sugieren que dichos costos podrían ser mayores que en los países desarrollados. ${ }^{16}$

En México no existe información sobre los costos de atención médica por el consumo de tabaco, y menos aún de su impacto económico en el sector salud. En este contexto, el presente estudio se plantea como objetivo principal estimar en la Delegación Morelos del IMSS los costos directos de la atención médica* (ambulatoria y hospitalaria) de tres enfermedades causadas por el consumo de tabaco: infarto agudo del miocardio (IAM), enfermedad pulmonar obstructiva crónica (EPOC) y cáncer de pulmón (CP).

\section{Materiales y métodos}

Las estimaciones de los costos de atención médica se realizaron desde la perspectiva del proveedor de servicios de salud, considerando los criterios y el enfoque de

\footnotetext{
* Costos directos de atención médica: se definen como el valor del consumo de todos los recursos, tanto humanos como materiales, que intervienen en el diagnóstico, tratamiento y rehabilitación de los pacientes con una enfermedad.
}

la metodología de costeo de enfermedad (COI). ${ }^{17} \mathrm{El}$ estudio se efectuó de octubre de 2001 a mayo de 2002, en las unidades de atención médica de niveles de atención $\mathrm{I}^{*}$ y II ${ }^{\ddagger}$ de la Delegación Morelos del IMSS.

\section{Estimación de costos de atención médica}

Utilizando la metodología de consenso de panel de expertos, ${ }^{18,19}$ se elaboraron tres guías diagnóstico-terapéuticas (GDT) ${ }^{20}$ con el fin de establecer los requerimientos necesarios para realizar el diagnóstico, la clasificación del grado de severidad y el tratamiento médico de cada una de las enfermedades en estudio, en los niveles de atención I y II de la delegación Morelos del IMSS. A partir de estas GDT se crearon dos "casos tipo" por enfermedad, considerando la utilización de servicios médicos en los dos niveles de atención de acuerdo con el grado de severidad de la enfermedad.

Para efectos del análisis de costos, la atención médica se agrupó en cinco eventos médicos ${ }^{\S}$ relevantes: a) atención ambulatoria, b) atención de urgencias, c) hospitalización, d) quirófano y e) atención en unidad especializada. Los costos unitarios de los eventos se estimaron teniendo en cuenta el modelo contable de "Presupuesto capitado" para el IMSS. ${ }^{21}$ Los costos de los materiales y medicamentos se estimaron con base en los precios de compras consolidadas de la institución para el año 2001. El costo del recurso humano se calculó con base en el salario integral anualizado considerando las prestaciones sociales y otros beneficios percibidos por el trabajador. Los costos de capital se depreciaron y

* Primer nivel de atención (prevención primaria): proporciona atención ambulatoria sin hospitalización. La atención médica de este nivel la realizan médicos familiares, profesionales de la salud (enfermeras y trabajadoras sociales) entrenados especialmente en servicios de medicina preventiva y curativa, así como en la promoción de la salud.

‡ Segundo nivel de atención (prevención secundaria): proporciona atención médica en las cuatro especialidades básicas (pediatría, cirugía, medicina interna, ginecología y obstetricia) y en algunas subespecialidades como cardiología, neurología, oncología clínica, entre otras. Estos servicios reciben apoyo de las áreas de anestesiología, laboratorio clínico, rayos X, trabajo social, nutrición, banco de sangre, farmacia y ambulancia). Además, apoyan el sistema de referencia y contrarreferencia de pacientes con los niveles I y III.

$\S$ Evento: conjunto de acciones desarrolladas en secuencia cronológica y funcional, que tienen la finalidad de otorgar un servicio específico de salud a los pacientes con alguno de los padecimientos en estudio. Estas acciones se pueden definir y medir y, por lo tanto, es posible asignarles un costo. Los exámenes de ayudas diagnósticas (laboratorio clínico, patología e imágenes diagnósticas) quedaron incluidos en cada uno de los eventos donde fueron solicitados. 
prorratearon de acuerdo con la metodología de costo anual equivalente (CAE). ${ }^{22,23}$

Los costos de los eventos médicos se anualizaron teniendo en cuenta la frecuencia de utilización de los recursos según lo recomendado por los paneles de expertos (anexo I, ecuaciones 1 y 2). Los costos totales de enfermedad se obtuvieron multiplicando el costo promedio anual de los casos* por el número de casos incidentes por grado de severidad registrados en la Delegación Morelos en el año 2001 (anexo I, ecuación 4).

La metodología para la atribución de costos al tabaquismo se basa en el concepto epidemiológico de la fracción atribuible a un factor de riesgo causal (FA); ésta se refiere al porcentaje de la enfermedad que podría reducirse si se eliminara el factor causal (tabaco) (anexo I, ecuaciones 5 y 6). ${ }^{24-26}$ En este estudio se utilizó la FA al consumo de tabaco para IAM previamente estimada por Salazar y colaboradores en la población derechohabiente de 35-65 años del IMSS, Morelos. ${ }^{27-28}$ Dado que en la actualidad no se cuenta con información de la FA al consumo de tabaco para EPOC y CP, se asumieron los valores estimados por el Centro de Control de Enfermedades de Estados Unidos de América (CDC). ${ }^{29}$

\section{Resultados}

\section{Infarto agudo del miocardio}

El costo promedio anual ${ }^{*}$ de un caso de IAM asciende a 79530 pesos, de los cuales $90 \%$ (70 517 pesos) corresponde a la atención inicial en el servicio de urgencias y al manejo en la unidad de cuidados intensivos (nivel de atención II). El 10\% restante (1 308 pesos) se destina a las acciones de seguimiento del paciente realizadas por el médico familiar en el nivel de atención I (cuadro I). Los costos totales anuales de atención médica ${ }^{\ddagger}$ del IAM en la delegación ascienden a 27 millones 199 mil 534 pesos, de los cuales se atribuyen al consumo de tabaco 15 millones 231 mil 739 pesos (cuadro II).

\section{Enfermedad pulmonar obstructiva crónica}

El costo promedio anual* de un caso de EPOC es de 73 303 pesos. En esta enfermedad en particular las estima- ciones de costos difirieron de manera importante según el grado de severidad de la enfermedad; así, el costo promedio anual de un caso de EPOC estable corresponde a 6628 pesos y el de un EPOC exacerbado a 139978 pesos (cuadro I). Esta marcada diferencia se debe a que los casos severos con exacerbaciones requieren no sólo de un manejo hospitalario prolongado, sino de oxigenoterapia permanente, a través del programa de atención domiciliaria del enfermo crónico, lo que representa un costo promedio anual de 66643 pesos. Los costos totales anuales de la atención médica ${ }^{\ddagger}$ de la enfermedad pulmonar obstructiva crónica en la delegación ascienden a 114 millones 645 mil 892 pesos, de los cuales 99 millones $741 \mathrm{mil}$ 926 pesos se atribuyen al consumo de tabaco (cuadro II).

\section{Cáncer de pulmón}

El costo promedio anual ${ }^{*}$ de un caso de $\mathrm{CP}$ es de $102 \mathrm{mil}$ 215 pesos, de los cuales $99 \%$ (102 mil pesos) corresponde a la atención hospitalaria especializada que se realiza en los servicios de oncología clínica y quimioterapia, con costo promedio anual de 58 mil pesos (cuadro I). Los costos totales anuales $\ddagger$ de la atención médica del cáncer de pulmón en la delegación ascienden a 10 millones 323 mil 745 pesos, de los cuales 8 millones 775 mil 184 pesos se atribuyen al consumo de tabaco (cuadro II).

\section{Costos atribuibles al consumo de tabaco}

Los costos de atención médica de estas tres enfermedades para la Delegación Morelos en los dos niveles de atención ascienden a 152 millones 169 mil 170 pesos al año, de los cuales 123 millones 748 mil 848 pesos se atribuyen directamente al consumo de tabaco (cuadro II). Este gasto corresponde a 7.3\% del presupuesto anual de la Delegación Morelos y podría ahorrarse si se implementaran medidas de prevención y control en la población trabajadora o se ofrecieran alternativas para abandonar el hábito tabáquico a la población derechohabiente de la Delegación.

\section{Discusión}

Los resultados que se presentan aquí corresponden al primer estudio realizado en México de costos de atención médica atribuibles al consumo de tabaco. Las es-

\footnotetext{
* Costo promedio anual por caso: corresponde a los costos promedios ponderados por la proporción de casos de enfermedad según el grado de severidad.

‡ Costo total anual de atención médica: corresponde a la atención médica en la Delegación Morelos, y considera el número de casos nuevos por enfermedad informados en el año 2001.
} 


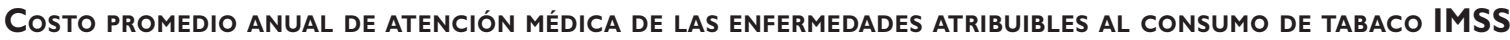
Morelos, $200 I^{*}$

\begin{tabular}{|c|c|c|c|c|c|c|}
\hline \multirow[b]{2}{*}{ Caso tipo $0^{\ddagger}$} & \multicolumn{2}{|c|}{ IAM } & \multicolumn{2}{|c|}{ EPOC } & \multicolumn{2}{|c|}{$C P$} \\
\hline & $\begin{array}{l}\text { IAM sin elevación } \\
\text { del segmento ST }\end{array}$ & $\begin{array}{l}\text { IAM con elevación } \\
\text { del segmento ST }\end{array}$ & $\begin{array}{l}\text { EPOC } \\
\text { estable }\end{array}$ & $\begin{array}{c}\text { EPOC } \\
\text { exacerbado }\end{array}$ & $\begin{array}{c}C P \\
\text { estadio IIB }\end{array}$ & $\begin{array}{c}C P \\
\text { estadio IV }\end{array}$ \\
\hline \multicolumn{7}{|l|}{ Atención médica } \\
\hline \multicolumn{7}{|l|}{ Nivel de atención I } \\
\hline \multicolumn{7}{|l|}{ Atención ambulatoria } \\
\hline Atención ambulatoria médico familiar & I 744 & 872 & 2180 & 654 & 218 & 218 \\
\hline Costo promedio nivel atención I & 1744 & 872 & 2180 & 654 & 218 & 218 \\
\hline \multicolumn{7}{|l|}{ Nivel de atención II } \\
\hline \multicolumn{7}{|l|}{ Atención ambulatoria } \\
\hline Consulta médica especializada* & 2369 & 4154 & 1790 & 1585 & 3897 & 15401 \\
\hline Atención urgencias & 2022 & 2022 & 2658 & 4389 & 1123 & 13840 \\
\hline Atención unidad cuidados intensivos ( $\mathrm{UCl}$ ) & 37509 & 61563 & & 61430 & & 13210 \\
\hline \multicolumn{7}{|l|}{ Atención hospitalaria } \\
\hline Hospitalización & 15338 & 16057 & & 50667 & 14712 & 31016 \\
\hline Atención domiciliaria enfermo crónico (ADEC) & & & & 15976 & & \\
\hline Quimioterapia & & & & & 28692 & 28692 \\
\hline \multicolumn{7}{|l|}{ Cirugía } \\
\hline Proc. quirúrgicos de diagnóstico & & & & 5277 & 5831 & 5143 \\
\hline Costo promedio nivel de atención II & 57238 & 83796 & 4448 & 139324 & 54255 & 107302 \\
\hline Costo promedio anual caso & 58982 & 84668 & 6628 & 139978 & 54473 & 107520 \\
\hline
\end{tabular}

\section{Cuadro II}

\section{Costos totales atribuibles al consumo de tabaco IMSS Morelos, 200 I*}

\begin{tabular}{|c|c|c|c|c|}
\hline Enfermedad & Costo promedio anualF & Casos incidentes & $\begin{array}{l}\text { Casos atribuibles } \\
\text { al consumo de tabaco }\end{array}$ & $\begin{array}{c}\text { Costos médicos atribuibles } \\
\text { al tabaco* }\end{array}$ \\
\hline Infarto agudo del miocardio & & & & 15231739 \\
\hline$\overline{\text { FA:056 }(0.45-0.66)^{\S}}$ & 79531 & 342 & $192(154-226)$ & (I2 $239790-1795 \mid 692)$ \\
\hline Enfermedad pulmonar obstructiva crónica & & & & 99741926 \\
\hline$\overline{\text { FA:087 }(0.81-0.91)^{\#}}$ & 73303 & 1564 & I 36| (| 267-I 423) & $(92863 \quad 173-104327762)$ \\
\hline Cáncer de pulmón & & & & 8775183 \\
\hline$\overline{\text { FA:085 }(0.72-090)^{\#}}$ & 102215 & 101 & $86(73-91)$ & (7 $433096-9291370)$ \\
\hline \multirow{2}{*}{\multicolumn{4}{|c|}{$\begin{array}{l}\text { Costos atribuibles al consumo } \\
\text { de tabaco IMSS Morelos 200I }\end{array}$}} & 123748848 \\
\hline & & & & $(112536059-131570825)$ \\
\hline
\end{tabular}


timaciones apenas permiten dimensionar la magnitud del impacto del tabaquismo en la atención médica en tan sólo una delegación de la mayor institución de servicios de salud del país: la carga económica del tabaquismo en el sector salud es únicamente una parte del costo social para la población mexicana.

La metodología de consenso de panel de expertos, a través de la elaboración de las GDT y de los casos tipo, así como de la experiencia clínica de los profesionales de la salud, ${ }_{,}^{30}$ permite establecer, de manera rápida y con poca inversión financiera, la utilización de servicios de atención médica para cada enfermedad en cada uno de sus niveles.

La importancia de desarrollar este tipo de estudios en México y los países en desarrollo radica en que no es posible extrapolar las estimaciones de los países desarrollados, ya que éstas dependen en gran medida de las características de la epidemia de tabaquismo y de las diferencias en el tratamiento de enfermedades en cuanto a eficacia, disponibilidad de servicios y acceso financiero. ${ }^{15}$

Estudios realizados en Estados Unidos de América de los costos de atención médica atribuibles al tabaco han estimado un costo anual que oscila entre 8.2 y 72.7 billones de dólares, el equivalente a un rango entre $0.46 \%$ y $1.15 \%$ del PIB. ${ }^{14,31-33}$ Esta variabilidad observada en las estimaciones se debe a las diferentes metodologías utilizadas por los autores; por lo tanto, se requiere cautela al tratar de realizar comparaciones entre un estudio y otro. No se debe perder de vista que las estimaciones de costos de atención médica pueden variar dependiendo de la perspectiva de costeo utilizada (proveedor de servicios de salud, paciente, social), las categorías de costos que se han considerado (costos directos de atención médica, costos por pérdida de productividad) y la perspectiva temporal seleccionada (transversal, longitudinal). Las estimaciones aquí presentadas se encuentran subestimadas ya que no contemplan la totalidad de enfermedades relacionadas con el consumo de tabaco ni los costos por pérdidas de productividad, que, según estudios internacionales, podrían llegar a ser tres veces más que los costos de atención médica. ${ }^{31-33}$ Dado que este estudio se realizó desde la perspectiva del IMSS, teniendo en cuenta la atención médica en el nivel de atención I y II durante el año posterior al diagnóstico del caso de enfermedad, los resultados son válidos y extrapolables únicamente a la población del IMSS de Morelos. Las principales limitaciones de este estudio se centran en que éste tiene un enfoque transversal, está limitado a un proveedor de servicios de salud y no contempla los costos indirectos que tienen gran impacto social.

Finalmente, para estimar la carga económica del consumo de tabaco en México, se recomienda reprodu- cir este estudio en el plano nacional, ampliar la estimación de los costos de atención médica directos (costos de atención médica y gasto de bolsillo) e indirectos (pérdidas de productividad y muerte prematura), así como considerar otras patologías relevantes relacionadas con el consumo de tabaco, como la enfermedad cerebro vascular, junto con aquéllas causadas por la exposición al humo de tabaco de segunda mano: enfermedades respiratorias en los niños, bajo peso al nacer, entre otras.

Este tipo de estudio permitirá contar con información local para priorizar el problema del tabaquismo dentro de la agenda de trabajo de la salud pública, agregará conocimiento para los países en desarrollo y proveerá información de primera mano para los tomadores de decisiones en la elaboración y puesta en práctica de políticas públicas para el control del tabaquismo en México. ${ }^{34}$

\section{Agradecimientos}

A los profesionales de la salud que participaron en el panel de expertos: Dra. Eloína Cordero, Dr. Ramón Acevedo, Dr. Fernando Téllez, Dr. Gerardo Araujo, Dra. Jesabel Cruz, Dra. Rosa María Muñoz, Lic. Margarita Menes, Lic. Feliz Ortiz, Lic. Graciela Mendiola; a los profesionales del área administrativa-financiera: CP María Elena Ovando, CP Amelia Guzmán, así como a todo el grupo de trabajo de educación médica, archivo, de las áreas administrativas del Hospital General Regional 01 de Cuernavaca, Morelos, y a las personas del área de informática de la Delegación Morelos del IMSS.

$\mathrm{Al}$ Centro Internacional Fogarty de los institutos nacionales de salud en los Estados Unidos de América por su apoyo a través del Grant Número R01-HL-73699 y al Consejo nacional de Ciencia y Tecnología (CONACyT) por el apoyo a través del Grant SEP-CONACYT $2002-140593$.

\section{Referencias}

I. U.S Department of Health, Education and Welfare (USDHEW). Smoking and Health. Report of the Advisory Committee to the Surgeon General of the Public Health Service. Washington, D.C: U.S. Government Printing office: DHEW publication No. (PHS) II 03, 1964. 2. U.S Department of Health and Human Services (USDHHS). The Health Consequences of Smoking: Cancer.A Report of the Surgeon General,Washington, D.C: U.S. Department of Health and Human Services, Public Health Service, Office on Smoking and Health, DHHS Publication No. (PHS) 82-50179, 1982.

3. U.S Department of Health and Human Services (USDHHS). The Health General. Rockville (MD): U.S. Department of Health and Human Services, Public Health Service, Office on Smoking and Health, DHHS Publication No. (PHS) 84-50204, 1983.

4. Great Britain Scientific Committee on Tobacco and Health Great Britain Department of Health Scientific Committee on Tobacco and 
Health. Report of the Scientific Committee on Tobacco and Health. Pitman Medical Publishing Co., LTD London, 1988.

5. Samet J. Los riesgos del tabaquismo activo y pasivo. Salud Publica Mex 2002; 44 (SI): SI 44-SI 60.

6. US Department of Health and Human Services (USDHHS). The health consequences of smoking:A Report of the Surgeon General.Atlanta (GA): US Department of Health and Human Services. Centers for Disease Control and Prevention. National Center for Chronic Disease Prevention and Health Promotion, Office on Smoking and Health, 2004. 7. The World Bank. Curbing the epidemic: Governments and Economics of Tobacco Control. Washington, DC:World Bank, 1999.

8. SSA-CONADIC. Encuesta Nacional de Adicciones 2002:Alcohol, tabaco y otras drogas. Resumen Ejecutivo, México, SSA-CONADIC, 2002

9. INEGI-SSA. Estadísticas de mortalidad en México: Muertes registradas en el año 2000. Salud Publica Mex 2002; 44 : 266-282.

10.Valdés-Salgado R. Las cifras de la epidemia. Daños a la salud y mortalidad atribuible. En: Primer informe sobre combate al tabaquismo. México ante el Convenio Marco para el Control del Tabaco. México. Cuernavaca: Instituto Nacional de Salud Pública, 2005:29-4I.

I I. Hodgson TA, Meiners MR. Cost-of-illness methodology:A guide to current practices and procedures. Milbank Mem Fund Q 1982; 60:429-462.

12. MacKenzie T, Bartecchi C, Schier R. The human costs of tobacco use. N Engl J Med 1994; 330: 975-980.

13. Barendregt J], Bonneu L,Van Der Mass PJ. The health care costs of smoking. N Engl J Med 1997; 337: 1052-1057.

14. Warner K, Hodgson TA, Carroll C. Medical cost of smoking in the United States: Estimates, their validity, and their implications. Tob control 1999; 8:290-300.

15. Leistikow B. The Human and Financial Costs of smoking. En: Smoking and Pulmonary and Cardiovascular Diseases. Clinics Chest Medicine 2000; 21:189-195.

16. Lightwood J, Collins D, Lapsley H, Novotny T. Estimating the costs of tobacco use. Tobacco control in developing countries. Editors Jha $P$ and Chaloupka F. Nueva York: Oxford University Press, 2000:63-99.

17. Single E, Collins D, Easton B, Harwood H, Lapsley H, Kopp P et al. International Guidelines for estimating the Costs of Substance Abuse. Second Edition. Toronto, 200I.

18. Jones J, Hunter D. Qualitative Research: Consensus methods for medical and health services research. BMJ 1995; 31 I:376-380.

19. Pagliari $C$ et al. The potential influence of small group processes on guide line development. J Eval Clin Pract 200I; 7: 165-173.
20. IMSS. Guías Diagnóstico-Terapéuticas: IAM, EPOC, CP. Cuernavaca, Morelos, 200I. (Documento interno del IMSS)

2I. IMSS. Dirección de Planeación y Finanzas. Coordinación de presupuesto, contabilidad y evaluación financiera. Metodología de costeo "Presupuesto Capitado", Instructivo para su uso. Ciudad de México, México, 2000. (Documento Interno del IMSS).

22. Drummond M, O'Brien B, Stoddart G, Torrance G. Métodos para la evaluación económica de los programas de asistencia sanitaria. Segunda edición. Madrid: Ediciones Díaz de Santos, 2001.

23. Castaño S. Cómo calcular sus costos. Guía para profesionales de la salud. Centro de Gestión Hospitalaria. Bogotá, Colombia, 1997. 24. RITC.At what cost? The economic impact of tobacco use on national health systems, societies and individuals: A summary of methods and findings. Monographs series No. I, Otawa, 2003. 25. Rothman K, Greenland S. Modern epidemiology; 2nd ed. Hagerstown: Lippincott-Raven, 1997

26. Szklo M, Nieto J. Epidemiology: Beyond the basics. Gaithersburg, Maryland:Aspen Publisher Inc, 2000.

27. Salazar E, Sánchez L, López L, Estrada A, Lazcano E, Hernández M. El tabaquismo y su fracción atribuible en la enfermedad isquémica cardiaca. Salud Publica Mex 2002; 44, (SI): S34-S43.

28. Kuritz S, Landis J.Attributable risk estimation from matched case control data. Biometrics 1998; 14 7:250-258.

29. CDC, Smoking-attributable mortality, morbidity and economic cost, SAMMEC [ sitio de internet] Disponible en: http://apps.nccd.cdc.gov/ sammec/login.asp.

30. Murphy MK et al. Consensus development methods and their use in clinical guideline development. Health Technol Assess 1998; 2 y 3.

31. Hodgson TA, Sinsheimer P, Browner W, Kopstein AN. The economic costs of the health effects of smoking, 1984. Rice DP. Milbank Q 1986; 64 (4):489-547.

32. Miller LS, Zhang X, Rice DP. State estimates of total medical expenditures attributable to cigarette smoking, 1993. Public Health Rep 1998; I I3: 447-458.

33. Miller VP, Ernst C, Collin F. Smoking-attributable medical care costs in the USA. Soc Sci Med 1999; 48: 375-391.

34. Caraballo R, Lee $C$. Consumo de tabaco entre los mexicanos y sus descendientes en Estados Unidos de América. Salud Publica Mex 2004; 46 (3): $241-250$. 
Anexo I

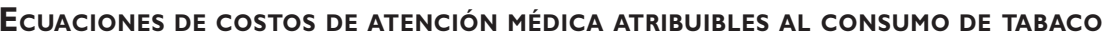

Ecuación I. Costos del evento médico

$$
C E_{m w k j i i}=\sum_{i=1}^{n_{3}} Q R_{m w k j i i} * P R_{m w k j i i}
$$

donde:

\begin{tabular}{|c|c|}
\hline$C E_{m w k j i l}=$ & Costo del evento médico de un paciente con una enfermedad de acuerdo con el grado de severidad \\
\hline$Q R_{\text {mwkjii }}=$ & $\begin{array}{l}\text { Utilización del recurso para la atención médica de un evento médico de un paciente con una enfermedad de acuerdo con el grado } \\
\text { de severidad }\end{array}$ \\
\hline$P R_{m w k j i i}=$ & $\begin{array}{l}\text { Precio o costo unitario del recurso para la atención médica de un evento de un paciente con una enfermedad de acuerdo con el } \\
\text { grado de severidad }\end{array}$ \\
\hline$m=$ & Enfermedad en estudio. (I. IAM, 2. EVC, 3. EPOC, 4. CP) \\
\hline$w=$ & Grado de severidad de la enfermedad (I. Leve, 2. Severo) \\
\hline$k=$ & Pacientes $\left(I, 2 \ldots n_{l}\right)$ \\
\hline$j=$ & $\begin{array}{l}\text { Tipo de evento médico (I. Atención ambulatoria, 2. Atención médica de urgencias, 3. Hospitalización, 4. Cirugía, 5. Atención en la } \\
\text { unidad de cuidados intensivos, 6. Quimioterapia, 7. Radioterapia) }\end{array}$ \\
\hline$I=$ & Número de eventos médicos (\#eventos/tipo de evento= $j)\left(1,2 \ldots n_{2}\right)$ \\
\hline$=$ & Recursos consumidos para la realización de la atención médica $\left(I, 2 \ldots n_{3}\right)$ \\
\hline
\end{tabular}

Ecuación 2. Costo promedio del evento médico

donde:

$$
A C E_{m w j}=\frac{\sum_{k=1}^{n_{1}} \sum_{1=1}^{n_{2}} C E_{m w k j i i}}{n_{m w j}}
$$

$A C E_{m w i}=\quad$ Costo promedio del evento médico

$C E_{\text {mwkili }}=\quad$ Costo del evento médico de un paciente con una enfermedad de acuerdo con el grado de severidad

$n_{m w j}=\quad \quad \quad$ Número de pacientes con la enfermedad según grado de severidad y evento

Ecuación 3. Costo promedio paciente de acuerdo con la severidad de la enfermedad

donde:

$$
A C C_{m w}=\frac{\sum_{k=1}^{n_{1}} \sum_{j=1}^{7} \sum_{l=1}^{n_{2}} C E_{m w k j i i}}{n_{m w}}
$$

$A C C_{m w}=\quad$ Costo promedio paciente de acuerdo con grado de severidad

$C E_{\text {mwkili }}=\quad$ Costo del evento médico de un paciente con una enfermedad de acuerdo con el grado de severidad

$n_{m w}=\quad$ Número de pacientes con la enfermedad según grado de severidad

Ecuación 4. Costo total de la enfermedad

donde:

$$
T C D_{m}=\sum_{w=1}^{2} \mathrm{ACC}_{m w} * N_{m w}
$$

$T C D_{m}=\quad$ Costo total de la enfermedad

$\mathrm{ACC}_{m \mathrm{w}}=\quad$ Costo promedio paciente de acuerdo con grado de severidad

$N_{m w}=\quad$ Casos incidentes de enfermedad de acuerdo con el grado de severidad

Ecuación 5. Fracción atribuible al consumo de tabaco

donde:

$$
S A F_{m}=\frac{p *(R R-I)}{p^{*}(R R-I)+I}
$$

$$
\begin{array}{ll}
S A F_{m}= & \text { Fracción atribuible al consumo de tabaco para la enfermedad } \\
p= & \text { Prevalencia de tabaquismo a nivel poblacional } \\
R R= & \text { Riesgo relativo de la enfermedad }
\end{array}
$$

Ecuación 6. Costos atribuibles al consumo de tabaco

donde:

$$
S A C_{m}=T C D_{m} * S A F_{m}
$$

$S A C_{m}=\quad$ Costos atribuibles al consumo de tabaco para la enfermedad

$T C D_{m}=\quad$ Costo total de la enfermedad

$S A F_{m}=\quad$ Fracción atribuible al consumo de tabaco para enfermedad determinada 